EUROPEAN ORGANIZATION FOR NUCLEAR RESEARCH

CERN-EP/2000-003

3 January 2000

\title{
PRODUCTION OF STRANGE AND MULTISTRANGE HADRONS IN NUCLEUS-NUCLEUS COLLISIONS AT THE SPS
}

\author{
Presented at "Quark Matter '99" by F. Antinori for the WA97 Collaboration:
}

F. Antinori ${ }^{5,9)}$, H. Bakke ${ }^{2)}$, W. Beusch ${ }^{5)}$, I.J. Bloodworth ${ }^{4)}$, R. Caliandro ${ }^{1)}$, N. Carrer ${ }^{9)}$, D. Di Bari ${ }^{1)}$, S. Di Liberto ${ }^{11)}$, D. Elia ${ }^{1)}$, D. Evans ${ }^{4)}$, K. Fanebust ${ }^{2)}$, R.A. Fini ${ }^{1)}$, J. Ftáčnik ${ }^{6)}$, B. Ghidini ${ }^{1)}$, G. Grella ${ }^{12)}$, H. Helstrup ${ }^{3)}$, A.K. Holme ${ }^{8)}$, D. Huss ${ }^{7)}$, A. Jacholkowski ${ }^{1)}$, G.T. Jones ${ }^{4)}$, J.B. Kinson ${ }^{4)}$, K. Knudson ${ }^{1)}$, I. Králik ${ }^{6)}$, V. Lenti ${ }^{1)}$, R. Lietava ${ }^{1)}$, R.A. Loconsole ${ }^{1)}$, G. Løvhøiden ${ }^{8)}$, V. Manzari ${ }^{1)}$, M.A. Mazzoni ${ }^{11)}$, F. Meddi ${ }^{11)}$, A. Michalon ${ }^{13)}$, M.E. Michalon-Mentzer ${ }^{13)}$, M. Morando ${ }^{9)}$, P.I. Norman ${ }^{4)}$, B. Pastirčák ${ }^{6)}$, E. Quercigh ${ }^{5)}$, G. Romano ${ }^{12)}$, K. Šafař́́k ${ }^{5)}$, L. Šándor ${ }^{5,6)}$, G. Segato ${ }^{9)}$, P. Staroba ${ }^{10)}$, M. Thompson ${ }^{4)}$, T.F. Thorsteinsen ${ }^{2)}$, G.D. Torrieri ${ }^{4)}$, T.S. Tveter ${ }^{8)}$, J. Urbán ${ }^{6)}$, O. Villalobos Baillie ${ }^{4)}$, T. Virgilii ${ }^{12)}$, M.F. Votruba ${ }^{4)}$ and P. Závada ${ }^{10)}$.

\begin{abstract}
We report the main results from experiment WA97 on the production of strange and multistrange hadrons at midrapidity in $\mathrm{Pb}+\mathrm{Pb}, \mathrm{p}+\mathrm{Pb}$ and $\mathrm{p}+\mathrm{Be}$ collisions at $158 \mathrm{~A} \mathrm{GeV} / c$. A comparison with some of the available models is presented.
\end{abstract}

\footnotetext{
1) Dipartimento I.A. di Fisica dell'Università e del Politecnico di Bari and Sezione INFN, Bari, Italy

2) Fysisk institutt, Universitetet i Bergen, Bergen, Norway

3) Høgskolen i Bergen, Bergen, Norway

4) School of Physics and Astronomy, University of Birmingham, Birmingham, UK

5) CERN, European Laboratory for Particle Physics, Geneva, Switzerland

6) Institute of Experimental Physics, Slovak Academy of Sciences, Košice, Slovakia

7) GRPHE, Université de Haute Alsace, Mulhouse, France

8) Fysisk institutt, Universitetet i Oslo, Oslo, Norway

9) Dipartimento di Fisica dell'Università and Sezione INFN, Padua, Italy

10) Institute of Physics, Academy of Sciences of the Czech Republic, Prague, Czech Republic

11) Dipartimento di Fisica dell'Università "La Sapienza" and Sezione INFN, Rome, Italy

12) Dipartimento di Scienze Fisiche "E.R. Caianiello" dell'Università and INFN, Salerno, Italy

13) Institut de Recherches Subatomiques, IN2P3/ULP, Strasbourg, France
} 



\section{INTRODUCTION}

The WA97 experiment was designed to study the production in nucleus-nucleus collisions of hadrons carrying one, two and three units of strangeness as a function of the centrality of the collision. This paper describes the main results obtained so far. These results are then compared with some model predictions.

The WA97 apparatus is briefly described in section 2. Section 3 deals with the measurement of the collision centrality. The results on the production of strange and multistrange hadrons are presented in sections 4 (yields) and $5\left(m_{T}\right.$ slopes). WA97 has isolated a sample of double $\mathrm{V}^{0}$ events; a first look at these data is offered in section 6 . A thorough comparison with the available models is now possible. We present some of our own work in this field in section 7 . Section 8 presents an outlook on the programme of the NA57 experiment, the successor of WA97, currently on the floor. We present our conclusions in section 9 .

\section{EXPERIMENTAL APPARATUS}

The WA97 set-up, sketched in figure 1, is described in $[1,2]$. The tracks emerging from the weak decays of strange hadrons were reconstructed in a Pixel Tracking Chamber (PTC) consisting of 7 planes of silicon pixel detectors with a pixel size of $75 \times 500 \mu \mathrm{m}^{2}$ and of 10 planes of silicon microstrip detectors with a $50 \mu \mathrm{m}$ pitch. The telescope had a cross section of $5 \times 5 \mathrm{~cm}^{2}$ and contained about $0.5 \times 10^{6}$ channels. The first plane of the PTC was placed $60 \mathrm{~cm}$ downstream of the target (90 cm for the proton beam reference runs), above the beam line and inclined (pointing to the target) in order to accept particles at central rapidity and medium transverse momentum. The phase space windows covered by the experiment for various particle species are given in [2]. The target and the PTC were placed inside the $1.8 \mathrm{~T}$ magnetic field of the CERN Omega magnet.

\section{pad chambers}

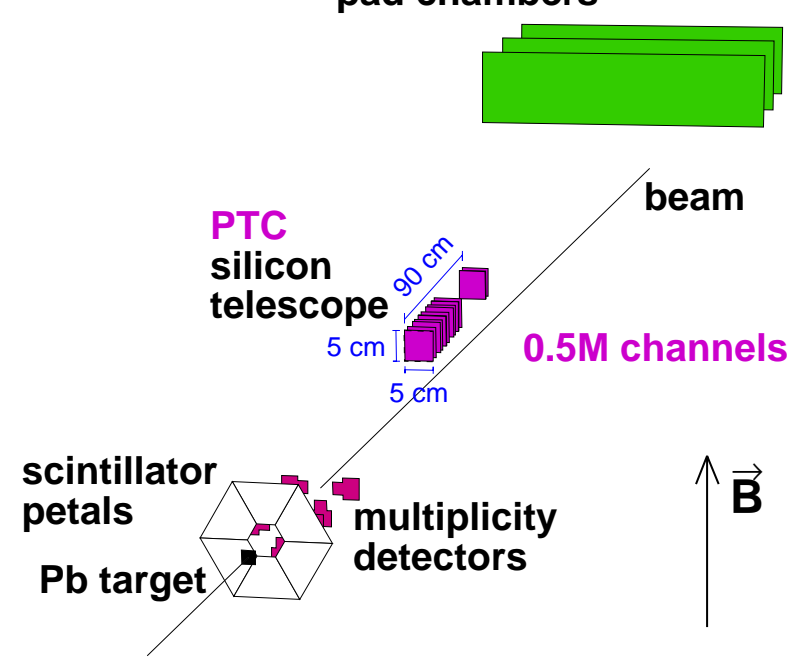

Figure 1: Sketch of the WA97 set-up.

The $158 \mathrm{~A} \mathrm{GeV/c}$ lead beam from the CERN SPS was incident on a lead target corresponding to $1 \%$ of the interaction length for the $\mathrm{Pb}$ beam. Scintillator detectors ("petals") placed behind the target provided an interaction trigger selecting the most central $\sim 40 \%$ of $\mathrm{Pb}+\mathrm{Pb}$ collisions. Two planes of microstrip multiplicity detectors (MSD) covering respectively the pseudorapidity region $2 \lesssim \eta \lesssim 3$ (station 1 ) and $3 \lesssim \eta \lesssim 4$ 

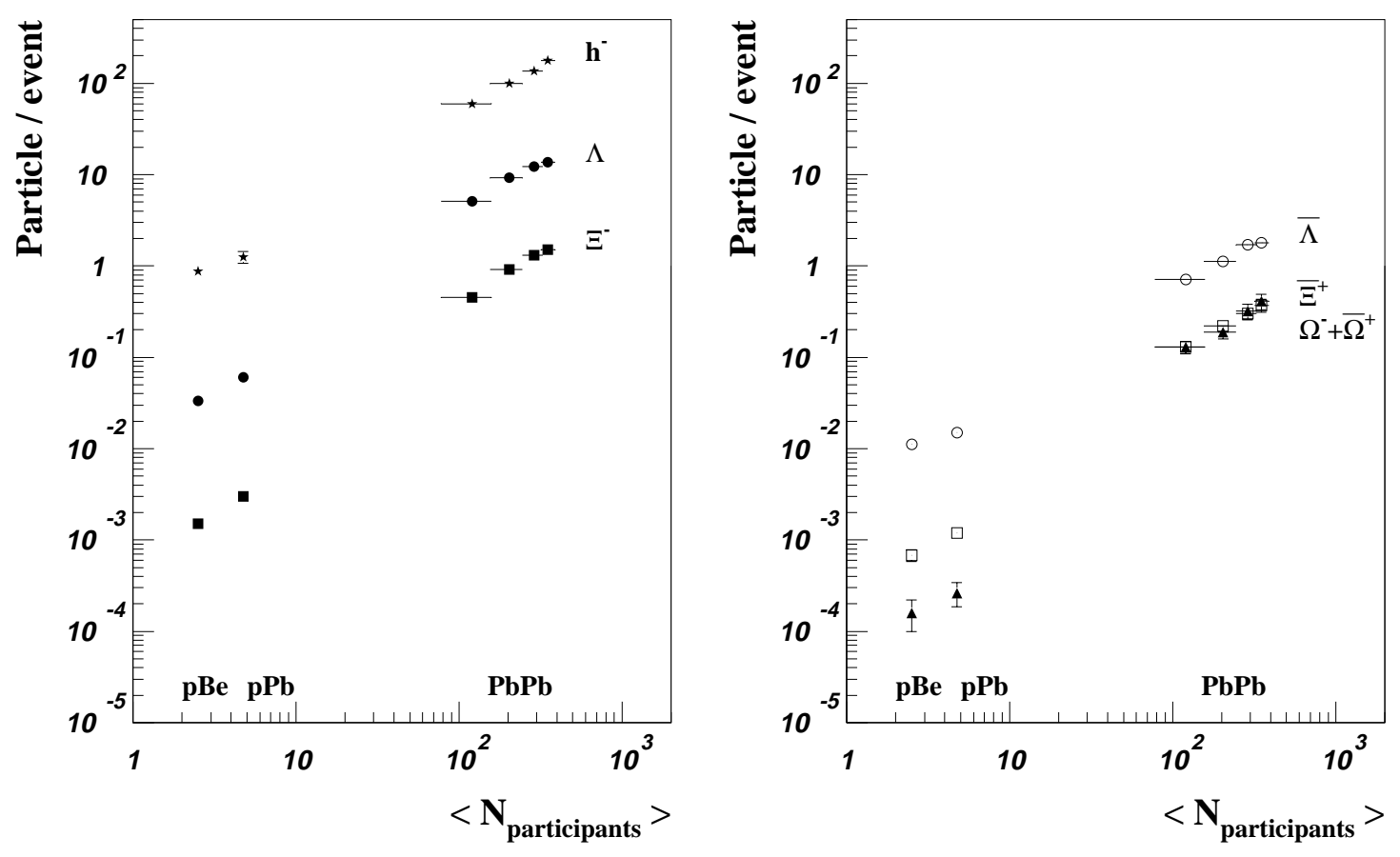

Figure 2: Yields per unit of rapidity at central rapidity as a function of the number of wounded nucleons for negative particles, $\Lambda$ and $\Xi^{-}$(left) and for $\bar{\Lambda}, \bar{\Xi}^{+}$and $\Omega^{-}+\bar{\Omega}^{+}$. See text for details.

(station 2), provided the multiplcity information on centrality which we used for the off-line study of the centrality dependence of particle yields.

In the proton reference runs, $\mathrm{Be}$ and $\mathrm{Pb}$ targets were used, both with a thickness corresponding to $8 \%$ of the interaction length. The protons in the $158 \mathrm{GeV} / \mathrm{c}$ positive beam were selected using two CERN Cherenkov CEDAR detectors [3]. In these runs, a trigger was applied demanding at least two tracks in the telescope, as required to find $\mathrm{V}^{0} \mathrm{~S}$ (two-track trigger). Data were also collected requiring at least one track in the telescope (one-track trigger). These data were used for the study of the production of negatives.

The details of the analysis, i.e. the reconstruction procedure, the extraction of the signals and the weighting procedures are described in $[2,4,5,6]$.

\section{CENTRALITY MEASUREMENT}

The centrality of the $\mathrm{Pb}+\mathrm{Pb}$ collisions is determined by analyzing the charged particle multiplicity measured in the interval $2<\eta<4$ by the multiplicity detector (MSD). The $\mathrm{Pb}+\mathrm{Pb}$ data sample is divided into four multiplicity classes and the average number of wounded nucleons $\left(N_{\text {participants }}\right)$ for each class is determined from a Wounded Nucleon Model [7] fit to the charged multiplicity distribution $\mathrm{d} \sigma / \mathrm{d} N_{c h}$. This analysis is discussed in detail in these proceedings [8].

The numbers of wounded nucleons corresponding to $\mathrm{p}+\mathrm{Pb}$ and $\mathrm{p}+\mathrm{Be}$ collisions have been determined as an average value for inelastic collisions in the framework of the Glauber model [9]. 

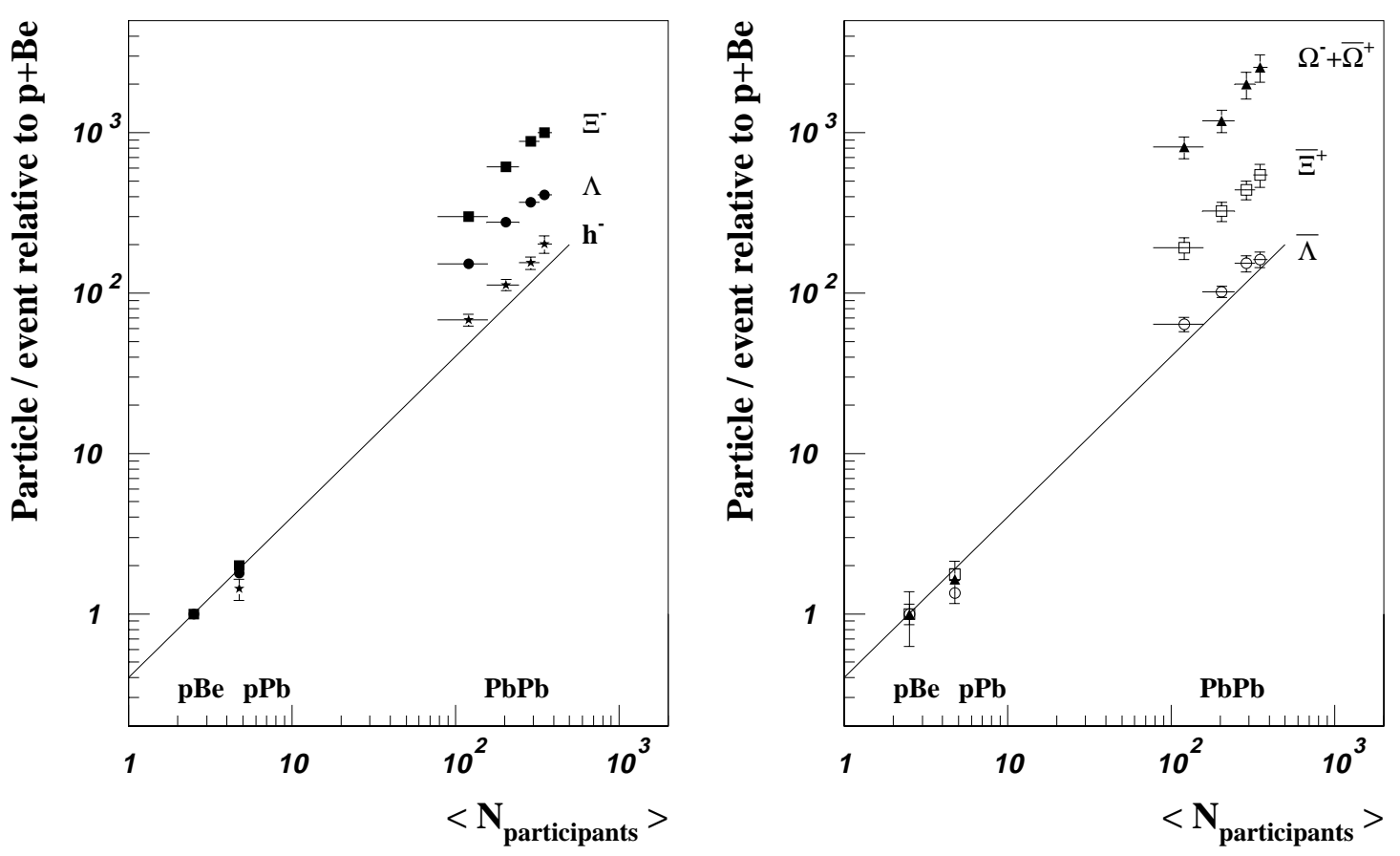

Figure 3: Yields per unit rapidity (same as in figure 2) relative to the $\mathrm{p}+$ Be yields.
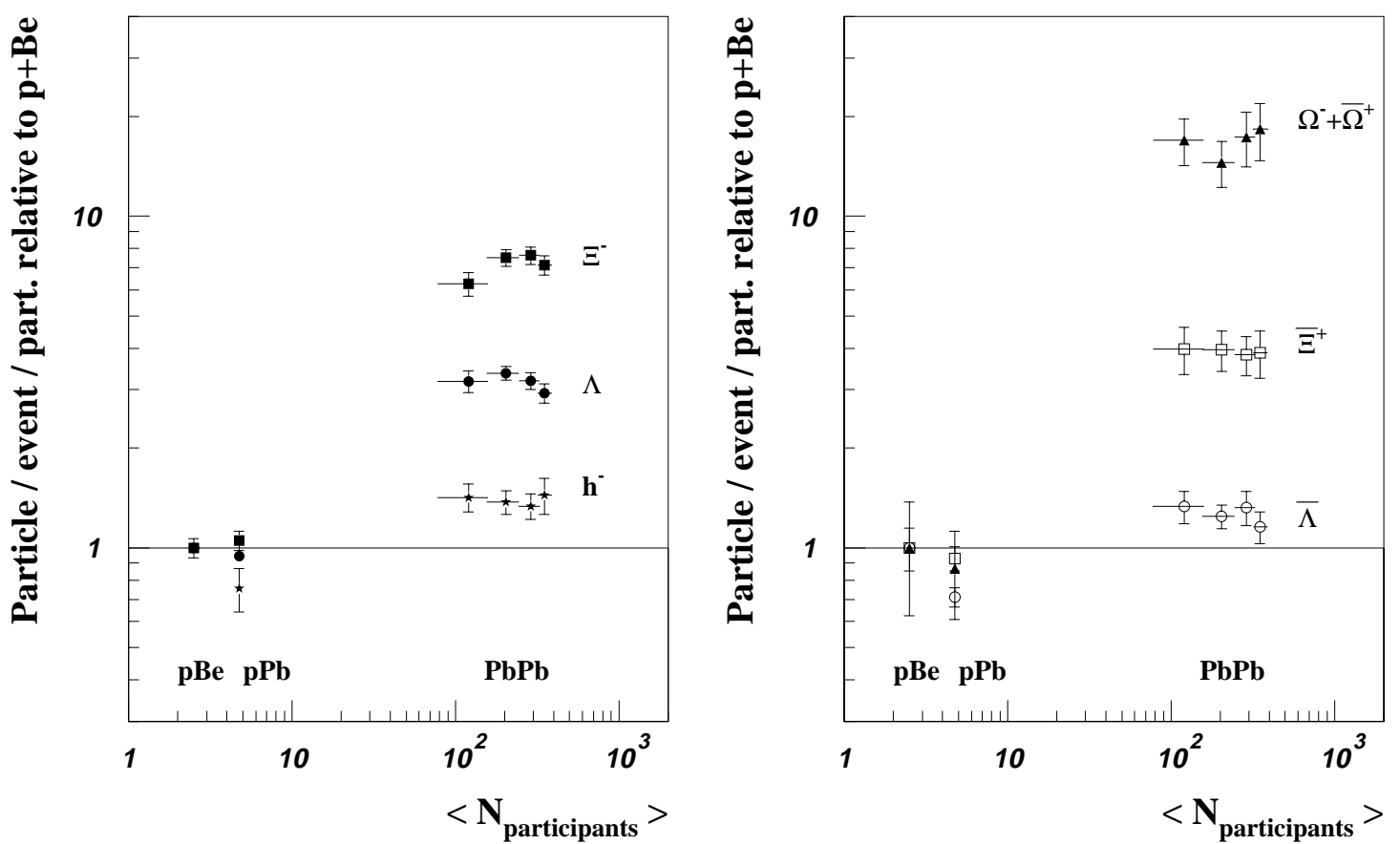

Figure 4: Yields per unit rapidity (same as in figure 2) per participant relative to $\mathrm{p}+\mathrm{Be}$. 


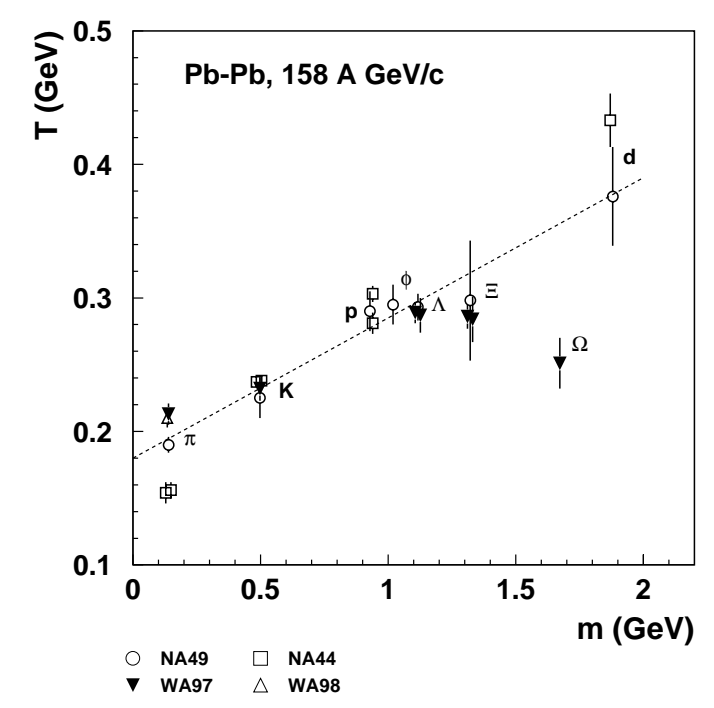

Figure 5: Dependence of the transverse mass inverse slope parameters on the particle rest mass.

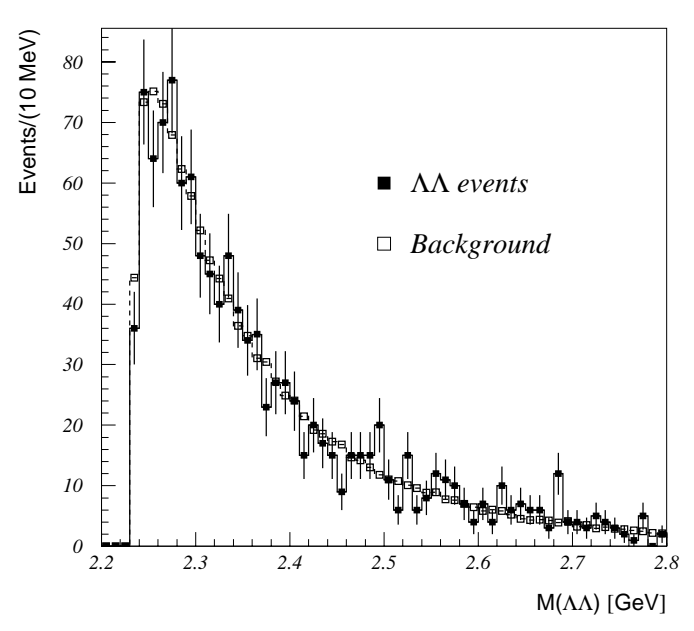

Figure 6: $\Lambda$ - $\Lambda$ invariant mass distribution for the data (1204 pairs) and the background obtained from mixing events of similar centrality, normalized to the number of $\Lambda-\Lambda$ pairs.

\section{PARTICLE YIELDS}

The yields per unit of rapidity at central rapidity for negative particles and for $\Lambda, \Xi^{-}, \Omega^{-}$and their antiparticles are plotted in figure 2 as a function of the average number of wounded nucleons for $\mathrm{p}+\mathrm{Be}$ and $\mathrm{p}+\mathrm{Pb}$ collisions and for the four centrality classes in $\mathrm{Pb}+\mathrm{Pb}$ collisions. All yields are extrapolated to a common phase space window covering full $p_{T}$ and one unit of rapidity centered at midrapidity. The horizontal error bars of the $\mathrm{Pb}+\mathrm{Pb}$ points represent the FWHM of the $N_{\text {participants }}$ distributions in the four multiplicity classes [8]. The recent $\mathrm{p}+\mathrm{Be}$ analysis is described in detail in these proceedings [10].

In figure 2, as well as in the following figures, particles are divided in two classes: those with at least one valence quark in common with the nucleon (plotted on the left) and those with no valence quark in common with the nucleon (plotted on the right). We have kept the two groups separate since it is empirically known that they may exhibit different production features (e.g. $\Lambda$ and $\bar{\Lambda}$ have different rapidity spectra both in $\mathrm{p}+\mathrm{S}$ and $\mathrm{S}+\mathrm{S}[11])$.

Figure 3 shows the yields relative to those measured in $\mathrm{p}+\mathrm{Be}$ collisions. The yields per participant relative to the $\mathrm{p}+\mathrm{Be}$ ones are shown in figure 4 . As can be seen from figures 3 and 4 , while the yields per participant have similar values in $\mathrm{p}+\mathrm{Be}$ and $\mathrm{p}+\mathrm{Pb}$ collisions for all the particles under study, all the yields per participant measured in $\mathrm{Pb}+\mathrm{Pb}$ are clearly above the $\mathrm{p}+\mathrm{A}$ values. The excess is larger for particles of higher strangeness content, up to a factor $\sim 20$ for $\Omega^{-}+\bar{\Omega}^{+}$. The yields per participant appear to be rather constant within the centrality range covered by WA97. This conclusion relies on the definition of participants as wounded nucleons [8].

\section{TRANSVERSE MASS SLOPE PARAMETERS}

The analysis of the transverse mass distributions is described in detail in these proceedings [12]. The $m_{T}$ distributions of negatives and strange particles were fitted to 


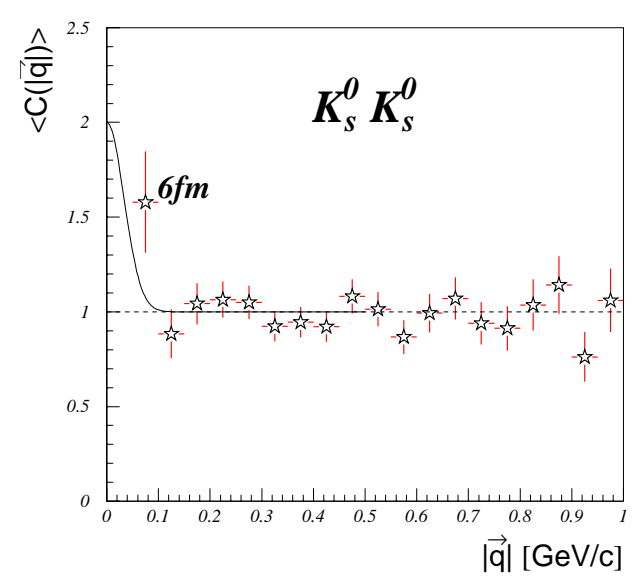

Figure 7: HBT relative momentum correlation of the $\mathrm{K}_{s}^{0}-\mathrm{K}_{s}^{0}$ pairs. The full curve shows the correlation expected for a fireball of a $6 \mathrm{fm}$ radius.

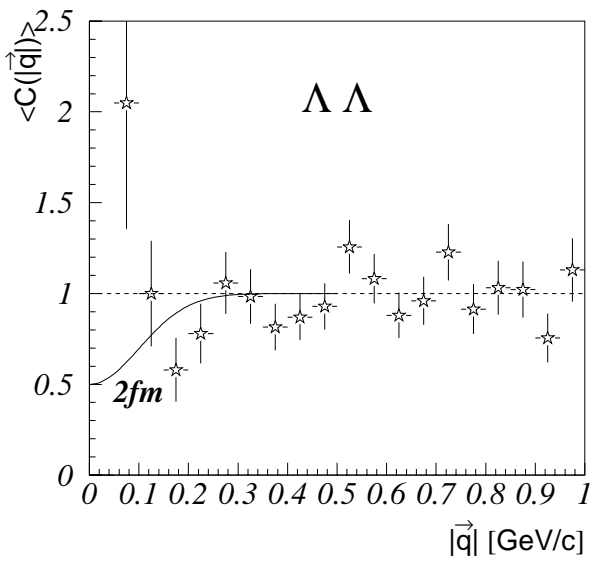

Figure 8: HBT relative momentum correlation of the $\Lambda-\Lambda$ pairs. The full curve shows the correlation expected for a fireball of $2 \mathrm{fm}$ radius.

the form

$$
\frac{\mathrm{d} N}{\mathrm{~d} m_{T}} \propto m_{T} \exp \left(-\frac{m_{T}}{T}\right) .
$$

The resulting values of the transverse mass slope parameter $T$ are plotted in figure 5 as a function of the rest mass of the particles, along with results from other CERN heavy-ion experiments (see e.g. [13] and references therein). The transverse mass slope parameters of multistrange particles deviate from the "standard" transverse flow expectation $[14,15]$, indicated by the straight line in figure 5. This is particularly visible in the case of the $\Omega$. We shall come back to this point in section 7 .

\section{DOUBLE V $\mathbf{V}^{0}$ EVENTS}

We have isolated a sample of events with two $\mathrm{V}^{0} \mathrm{~s}$ reconstructed in the PTC. The results presented here are discussed in detail in [16]. They were obtained on one half of the WA97 statistics and should correspond to about a quarter of the final statistics including NA57 data (see section 8).

In particular, we have 1204 events containing a $\Lambda-\Lambda$ pair. The $\Lambda-\Lambda$ mass spectrum for this sample is shown in figure 6 , together with a background sample constructed by mixing $\Lambda$ s from different events. There is no evidence of $\Lambda-\Lambda$ resonances. The probability that the two distributions are random samples of the same parent distribution has been calculated to be 0.49 by a Kolmogorov test.

We have had a first look at the two-particle correlation for $\mathrm{K}_{s}^{0}$ and $\Lambda$, by calculating the correlation function $C(|\vec{q}|)$, where $\vec{q}$ is the three-momentum difference of the pair in the collision center-of-mass system, according to a prescription described in [17]. The correlation functions for $1999 \mathrm{~K}_{s}^{0}-\mathrm{K}_{s}^{0}$ pairs and for the $1204 \Lambda-\Lambda$ pairs are plotted in figures 7 and 8 respectively. The curves superimposed to the plots show the expectation for the correlation function for a radius of $6 \mathrm{fm}$ and for a radius of $2 \mathrm{fm}$ respectively for $\mathrm{K}_{S}^{0}$ and $\Lambda$.

No firm conclusions are possible with the present statistics. 


\section{COMPARISON WITH MODELS}

WA97 has produced a comprehensive set of results on the production of strange particles in $\mathrm{p}+\mathrm{A}$ and $\mathrm{Pb}+\mathrm{Pb}$ collisions. These data should now allow thorough checks of the particle production models to be performed. In the following of this section, we shall comment on the comparison of our results with some of the available models.

A quark coalescence model has been recently proposed by A. Białas [18], and has subsequently been refined by J.Zimanyi and collaborators [19]. The model assumes that hadrons emerge from the statistical hadronization of uncorrelated quarks. In this case, it can be easily shown that simple relations should hold between the different antihyperon/hyperon production ratios. Such relations are seen to hold for the case of $\mathrm{Pb}+\mathrm{Pb}$ collisions, but they fail for $\mathrm{p}+\mathrm{Pb}$. This suggests that the quark degrees of freedom become relevant in the description of $\mathrm{Pb}+\mathrm{Pb}$ collisions. Such a coalescence scenario would be valid for particles emerging from a deconfined system, but it does not require thermalization.

When analysing particle production ratios, it is customary to fit the data in the framework of a thermal model. Recent examples of this type of analysis can be found in [20, 21]. It appears from such analyses that most particle ratios in $\mathrm{Pb}+\mathrm{Pb}$, including those involving multistrange hyperons and antihyperons, are close to their chemical equilibrium values. While in principle thermal models do not provide an insight on the history of the system before equilibration, the question remains how the abundances of rare particles such as $\Omega$ and $\bar{\Omega}$ can get close to their chemical equilibrium values within the short duration of a heavy-ion collision (a few $\mathrm{fm} / \mathrm{c}$ ).

We now turn to the comparison with some of the microscopic dynamical models. Most of the following comments are based on the work contained in a recent paper of ours [22], where RQMD 2.3 and VENUS 4.12 events have been simulated through a GEANT description of the WA97 apparatus, including a simulation of the trigger. We have checked the predictions of the two event generators against the data on the yields measured in $\mathrm{p}+\mathrm{Pb}$ and $\mathrm{Pb}+\mathrm{Pb}$ collisions, on their dependence on the number of participants, on the enhancement factors for the different species of strange and multistrange hadrons, and on the transverse mass slope parameters.

An example from this work is shown in figure 9, where the absolute values of the yields measured by WA97 in $\mathrm{p}+\mathrm{Pb}$ and $\mathrm{Pb}+\mathrm{Pb}$ collisions are compared with the predictions from the generators. The comparison shows that VENUS 4.12 overestimates the yields of multistrange hadrons both for the $\mathrm{p}+\mathrm{Pb}$ and the $\mathrm{Pb}+\mathrm{Pb}$ system. RQMD 2.3 overestimates the $\bar{\Omega}$ yields in $\mathrm{p}+\mathrm{Pb}$ and underestimates the yields of both $\Omega$ and $\bar{\Omega}$ in $\mathrm{Pb}+\mathrm{Pb}$ by about a factor 2 .

As for the transverse mass distributions, RQMD 2.3 succeeds in reproducing the behaviour of the inverse slope parameter $T$ versus the mass in $\mathrm{Pb}+\mathrm{Pb}$ collisions even for multistrange hadrons. It also succeeds in reproducing the inverse slope parameters observed in $\mathrm{p}+\mathrm{Pb}$ collisions.

In a recent paper [23] which compares the transverse mass slope parameters of strange particles in $\mathrm{Pb}+\mathrm{Pb}$ collisions with the predictions of RQMD 2.3, the deviation of multistrange particles from the "standard" flow curve is attributed to the fact that these hadrons, owing to their low cross sections for reinteraction in the hadronic medium, decouple rather early from the evolution of the hadronic system, and therefore are less affected by flow.

A similar picture emerges from a recent work by A.Dumitru et al. [24] using a model which employs hydrodynamics for the QGP phase and the UrQMD program for the transport after hadronization. 

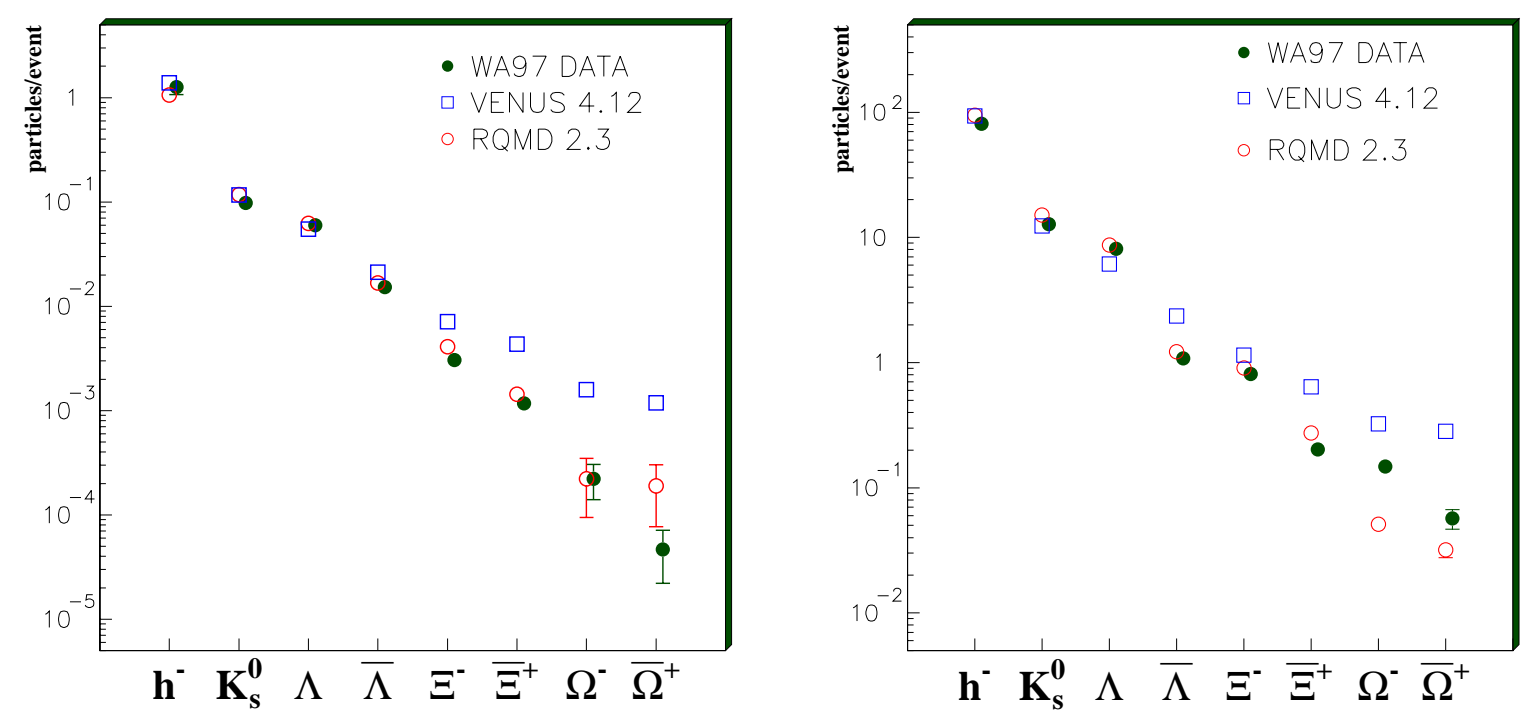

Figure 9: Particles per $\mathrm{p}+\mathrm{Pb}$ interaction (left) and per $\mathrm{Pb}+\mathrm{Pb}$ interaction (right) measured by WA97 (black circles) compared with the predictions of VENUS (open squares) and RQMD (open circles).

Other models ([25], DPM and [26], Hijing $B \bar{B}$ ) introduce mechanisms which increase the stopping of baryons. In both cases, additional mechanisms must be introduced in order to account for the measured production of multistrange hyperons: final state reinteractions [25] and ropes [26]. These models should be checked against the full set of available data, including transverse mass slope parameters, negatives, antihyperon to hyperon ratios, the behaviour with centrality, $\mathrm{p}+\mathrm{A}$ data.

Summarizing, thermal analyses suggest that the abundances of multistrange hyperons and antihyperons produced in $\mathrm{Pb}+\mathrm{Pb}$ collisions are close to chemical equilibrium. This is intriguing, since the total duration of the collision is estimated to be too short to allow equilibration of $\Xi$ and $\Omega$ by purely hadronic means.

The dynamical models VENUS 4.12 and RQMD 2.3 fail in describing the abundances of multistrange hadrons. As for the transverse mass slope parameters, RQMD 2.3 qualitatively reproduces the behaviour of multistrange particles as a result of an early decoupling from the rest of the hadronic fireball. DPM and HIJING $B \bar{B}$ need to be tested on the complete set of measured quantities.

\section{OUTLOOK}

The successor of WA97, experiment NA57, is currently on the floor [28]. The apparatus is rather similar to that of WA97, although a different magnet is used.

The main goals of NA57 are to extend the centrality range of WA97 for $\mathrm{Pb}+\mathrm{Pb}$ collisions at $158 \mathrm{~A} \mathrm{GeV/c} \mathrm{(1998} \mathrm{run)} \mathrm{and} \mathrm{to} \mathrm{measure} \mathrm{the} \mathrm{pattern} \mathrm{of} \mathrm{multistrange} \mathrm{hyperon}$ production in $\mathrm{Pb}+\mathrm{Pb}$ collisions at $40 \mathrm{~A} \mathrm{GeV} / c$ (1999 run), in order to search for an onset of the strangeness enhancement effect at lower values of the energy density.

In 1998, $230 \mathrm{M} \mathrm{Pb}+\mathrm{Pb}$ events were collected at $158 \mathrm{~A} \mathrm{GeV} / c$, essentially doubling the statistics collected by WA97. The new sample should allow to extend the centrality coverage down to about 50 wounded nucleons. 


\section{CONCLUSIONS}

WA97 has produced a comprehensive set of data on the production of multistrange hadrons in $\mathrm{p}+\mathrm{A}$ and $\mathrm{Pb}+\mathrm{Pb}$ collisions as a function of the number of wounded nucleons. This should allow to constrain the available models in detail.

The emerging picture is that the production of strange particles is enhanced when going from $\mathrm{p}+\mathrm{A}$ to $\mathrm{Pb}+\mathrm{Pb}$ collisions. The enhancement effect increases with the strangeness content of the particle up to a factor 20 for $\Omega+\bar{\Omega}$. This type of behaviour was predicted as a consequence of the QGP phase transition[27].

The mechanism responsible for the enhancement appears to be already saturated at $\mathrm{N}_{\text {participants }}=100$.

The behaviour of the transverse mass slope parameters for multistrange hadrons suggests that these particles decouple early from the hadronic phase. This would indicate that the increase in their abundance occurs rapidly in the early stages of the collision.

This picture is compatible with the expectations from a deconfinement scenario.

New data coming from NA57 for an extended centrality range and at a lower beam energy should allow a more stringent test of this hypothesis.

We would like to close this paper with a call for pre-dictions regarding the strangeness enhancement pattern for $N_{\text {participants }}<100$ at both 158 and $40 \mathrm{~A} \mathrm{GeV/c}$ beam momentum.

\section{References}

[1] F. Antinori et al., Nucl. Phys. A590 (1995) 139c.

[2] E. Andersen et al., Phys. Lett. B433 (1998) 209.

[3] C. Bovet et al., CERN Yellow Report CERN 82-12 (1982).

[4] R. Lietava et al. (WA97 Collaboration), in Proceedings of the 4th International Conference on Strangeness in Quark Matter, Padova, Italy, July 1998, J. Phys. G: Nucl. Part. Phys. 25 (1999) 181.

[5] R. Caliandro et al. (WA97 Collaboration), in Proceedings of the 4th International Conference on Strangeness in Quark Matter, Padova, Italy, July 1998, J. Phys. G: Nucl. Part. Phys. 25 (1999) 171.

[6] E. Andersen et al., Phys. Lett. B449 (1999) 401.

[7] A. Białas, M. Bleszyński and W. Czyż, Nucl. Phys. B111 (1976) 461.

[8] N. Carrer et al. (WA97 and NA57 Collaborations), these proceedings.

[9] C.Y. Wong, Introduction to High-Energy Heavy-Ion Collisions (World Scientific Publishing, Singapore, 1994) 251-264.

[10] D. Elia et al. (WA97 Collaboration), these proceedings.

[11] T. Alber et al., Z. Phys. C46 (1994) 195.

[12] L. Sandor et al. (WA97 Collaboration), these proceedings.

[13] J. Stachel, nucl-ex/9903007.

[14] I.G. Bearden et al., Phys. Rev. Lett. 78 (1997) 2080.

[15] G. Roland et al. (NA49 Collaboration), Nucl. Phys. A638 (1998) 91c.

[16] A. Jacholkowski et al. (WA97 Collaboration), in Proceedings of the 4th International Conference on Strangeness in Quark Matter, Padova, Italy, July 1998, J. Phys. G: Nucl. Part. Phys. 25 (1999) 423.

[17] C. Greiner and B. Müller, Phys. Lett. B219 (1989) 199.

[18] A. Białas, Phys. Lett. B442 (1998) 449.

[19] J. Zimanyi et al., hep-ph/9904501.

[20] P. Braun-Munzinger et al., nucl-th/9903010. 
[21] J. Rafelski and J. Letessier, nucl-th/9902365.

[22] F. Antinori et al., subm. to Eur. Phys. J. C.

[23] H. van Hecke, H. Sorge and N. Xu, Phys. Rev. Lett. 81 (1998) 5764.

[24] A. Dumitru et al., nucl-th/9901046.

[25] A. Capella and C.A. Salgado, hep-ph/9903414.

[26] S. Vance and M. Gyulassy, nucl-th/9901009.

[27] J. Rafelski, Phys. Lett. B262 (1991) 333.

[28] V. Manzari et al. (NA57 Collaboration), these proceedings. 